\title{
Associação entre excesso de peso e consumo de feijão em adultos
}

\author{
Association between overweight and \\ intake of beans among adults
}

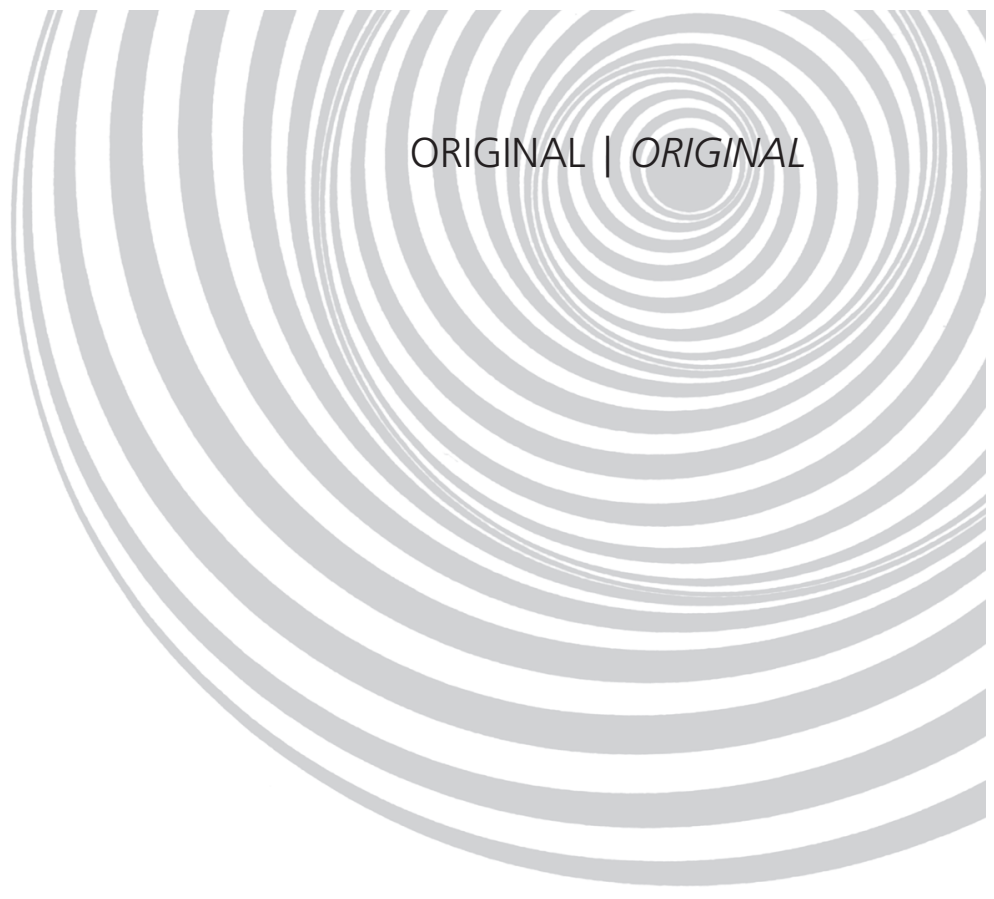

Sara Araújo SILVA

Priscilla de Nazaré Silva dos SANTOS 1

Erly Catarina MOURA ${ }^{1}$

RES U M O

\section{Objetivo}

Avaliar associação entre excesso de peso e consumo de feijão em adultos.

\section{Métodos}

O estudo constou de indivíduos adultos ( $\geq 18$ anos), moradores em Belém (PA), em 2005. A amostragem foi realizada por sorteio de residências com telefone fixo e de um morador adulto de cada casa sorteada. A variável desfecho foi excesso de peso, a variável explanatória consumo de feijão e as variáveis de controle foram idade, escolaridade e situação conjugal, além de atividade física no lazer e hábitos alimentares de risco. A análise dos dados foi feita pelo teste do qui-quadrado e por regressão logística.

\section{Resultados}

Foram avaliados 2352 indivíduos (39,8\% do sexo masculino). O excesso de peso atingiu mais os homens, $49,3 \%$, do que as mulheres, 34,0\% $(p<0,001)$. A prevalência de excesso de peso apresentou associação direta com idade em ambos os sexos e com escolaridade para homens, para as mulheres a associação com a escolaridade foi inversa. A variável referente ao consumo alimentar que melhor se associou com excesso de peso foi o consumo de feijão. Após ajuste para as demais variáveis, o risco de excesso de peso foi cerca de 1,4 vez maior para os homens que consomem feijão menos do que cinco vezes na semana, porém o inverso para as mulheres.

\section{Conclusão}

Os resultados indicam a necessidade de estudos mais controlados para melhor entendimento da associação entre consumo de feijão e excesso de peso.

Termos de indexação: Adultos. Consumo de feijão. Excesso de peso. Hábitos alimentares.

\footnotetext{
${ }^{1}$ Universidade Federal do Pará, Instituto de Ciências da Saúde, Faculdade de Nutrição. Av. Generalíssimo Deodoro, 1, Umarizal, 66050-060, Belém, PA, Brasil Correspondência para/Correspondence to: S.A. SILVA. E-mail: <silvanut@gmail.com>.
} 
240 S.A. SILVA et al.

\section{A B S T R A C T}

\section{Objective}

The objective of this study was to assess the association between overweight and intake of beans in adults.

\section{Methods}

The study population was 2,352 adults ( $\geq 18$ years of age) living in Belém (PA), Brazil, in 2005. Sampling was done by randomly selecting households with a telephone landline and then selecting an adult in the household. The dependent variable was overweight, the explanatory variable was intake of beans and the confounding variables were age, education level, marital status, leisure-time physical activity and risky food habits. The data were analyzed by the chi-square test and logistic regression.

\section{Results}

A total of 2,352 individuals were assessed where 39.8\% were males. The prevalence of overweight was higher in men $(49.3 \%)$ than in women $(34.0 \%$, p 0.001$)$. Overweight was directly associated with age in both genders and with education level in men. In women, overweight was inversely associated with education level. The food behavior variable that best associated with overweight was intake of beans. After adjustment for the other variables, the risk of overweight was approximately 1.4 times greater in men who ate beans less than five times per week, but the inverse was true for women.

\section{Conclusion}

The data show that better controlled studies are needed to understand the association between intake of beans and excess weight.

Indexing terms: Adults. Intake of beans. Overweight. Food habits.

\section{N T R O D U ÇÃ O}

Segundo a Organização Mundial da Saúde (OMS), mais de um bilhão de pessoas no mundo apresentam excesso de peso e esse número poderá chegar a 1,5 bilhão no ano de 2015, o que significa um aumento de $50 \%$, se a tendência atual de crescimento persistir, o que consequentemente acarretará maior risco de doenças cardiovasculares ${ }^{1}$.

No Brasil, dados de $2006^{2}$ mostram que 43,0\% da população adulta das capitais apresentam excesso de peso, atingindo mais os homens $(47,3 \%)$ do que as mulheres (38,8\%). Esse valor configura um aumento de 6,2\% em homens e $7,2 \%$ em mulheres quando comparados aos dados da Pesquisa de Orçamentos Familiares (POF), realizada em 2002 e 2003³, o que acarretará maior risco para as doenças crônicas não-transmissíveis, que representam, por sua vez, quase $50 \%$ dos óbitos atuais 4 .

Vários estudos demonstram associação entre aumento de peso e determinados comportamentos alimentares, como, por exemplo, o con- sumo excessivo de refrigerantes ${ }^{5}$ ou de bebidas alcoólicas $^{6}$. No Brasil7, observou-se que a dieta tradicional do brasileiro, baseada no arroz e no feijão como alimentos majoritários, associa-se a menor risco de excesso de peso em adultos. Todavia a situação torna-se mais preocupante pela constatação da mudança do hábito alimentar do brasileiro, caracterizada pela redução do consumo relativo de energias provenientes de cereais, feijão, raízes e tubérculos entre 1960 e $1980^{\circ}$, e crescente participação relativa de energias provenientes de açúcar refinado e refrigerantes entre 1988 e 1996. Recentemente, a Pesquisa Mundial da Saúde mostrou que $41 \%$ dos brasileiros adultos relataram consumir diariamente frutas, enquanto que menos de um terço (30\%) referiram o consumo diário de hortaliças ${ }^{10}$.

Levy-Costa et al. ${ }^{11}$, em estudo sobre distribuição e evolução da disponibilidade domiciliar de alimentos no Brasil entre os anos de 1974 e 2003, verificaram declínio no consumo de alimentos básicos e tradicionais da dieta do brasileiro, como o arroz e o feijão; aumento no consumo de produtos industrializados, como biscoitos e refri- 
gerantes; consumo excessivo de açúcar; insuficiente de frutas e hortaliças e aumento sistemático de gorduras em geral, resultados que apontam para tendências desfavoráveis do padrão alimentar, sobretudo do ponto de vista do excesso de peso.

Visto que o consumo alimentar está intimamente relacionado com a prevalência do excesso de peso, o presente estudo pretende avaliar a associação entre excesso de peso e consumo de feijão em adultos residentes na cidade de Belém (PA).

\section{MÉ T O D O S}

Este estudo é parte de um projeto multicêntrico - Sistema de Monitoramento de Fatores de Risco para Doenças Crônicas Não-Transmissíveis por meio de Entrevistas Telefônicas (SIMTEL) - que foi desenvolvido em cinco macro-regiões brasileiras, sendo a cidade de Belém (PA) selecionada para representar a região Norte. Trata-se de um estudo transversal realizado junto à população com 18 ou mais anos de idade, residente em moradias com pelo menos um telefone fixo em 2005.

Para estimar a frequência de qualquer fator com coeficiente de confiança de $95 \%$ e erro máximo de cerca de dois pontos percentuais, um mínimo de 2 mil entrevistas foram estipuladas. Em Belém, foram chamados 3500 números de telefone, sendo 2918 considerados elegíveis, isto é, potencialmente residenciais. Foram concluídas 2620 entrevistas, resultando numa taxa de sucesso de $89,9 \%$, com uma taxa de recusa de $7,0 \%$. Maiores detalhes metodológicos podem ser vistos em Monteiro et al. ${ }^{12}$.

Para este estudo, foram excluídos os indivíduos que não informaram peso e/ou altura e as mulheres grávidas, constituindo uma amostra de 2352 entrevistas.

A entrevista foi realizada por telefone, com o auxílio de um questionário eletrônico que permitia a leitura das questões e o registro imediato das respostas. As questões abordavam: a) caracte- rísticas demográficas e socioeconômicas dos indivíduos; b) características do padrão de alimentação (por exemplo: frequência do consumo de feijão, frutas e hortaliças e de alimentos fonte de gordura saturada e de bebidas alcoólicas), c) características indicativas da composição corporal (peso e altura recordados) e d) atividade física no lazer, entre outros.

Excesso de peso foi considerado variável desfecho e consumo de feijão variável explanatória. As demais variáveis independentes (idade, escolaridade, situação conjugal) foram consideradas de confusão. Adicionalmente, foram considerados atividade física no lazer e hábitos alimentares de risco, como baixo consumo de frutas, legumes e verduras, alto consumo de refrigerante e de bebidas alcoólicas e consumo de alimentos com gorduras saturadas.

Excesso de peso foi classificado conforme o Índice de Massa Corporal (IMC) em presente (IMC $\geq 25 \mathrm{~kg} / \mathrm{m}^{2}$ ) ou ausente (IMC $<25 \mathrm{~kg} / \mathrm{m}^{2}$ ), segundo a recomendação da Organização Mundial de Saúde ${ }^{13}$, de acordo à auto-referência ao peso e altura atuais.

O consumo de feijão foi classificado em três categorias: $\geq$ cinco dias por semana, três a quatro dias por semana e $\leq$ dois dias por semana, em reposta à questão: "Em quantos dias da semana o(a) sr(a) come feijão?"

Para atividade física no lazer, foram levantadas as seguintes questões: "O(a) sr(a) faz ou pratica algum tipo de exercício físico ou esporte? Qual o tipo principal de exercício físico ou esporte que o(a) sr(a) pratica? Quantos dias por semana o(a) sr(a) costuma praticar esporte ou exercício? No dia em que o(a) sr(a) pratica exercício ou esporte, quanto tempo dura essa atividade?" Considerou-se atividade física, no lazer, o indivíduo que relatou praticar atividade vigorosa pelo menos 20 minutos por dia, em pelo menos três dias da semana ou atividade moderada ou leve em pelo menos 30 minutos por dia em pelo menos cinco dias da semana ${ }^{14}$.

Foi considerado hábito alimentar de risco: baixo consumo de frutas, legumes e verduras $(<5$ 
dias por semana), alto consumo de refrigerante ( $\geq 3$ dias por semana) e de bebidas alcoólicas ( $>1$ dose por dia para mulher no último mês e $>2$ para homem), e hábito de consumo de alimentos com gorduras saturadas (leite integral e carne com gordura ou frango com pele). Para compor a variável baixo consumo de frutas, legumes e verduras, utilizou-se a combinação da resposta negativa para esses alimentos na forma in natura ou cozida, conforme as questões: "O(a) sr(a) costuma comer frutas todos ou quase todos os dias? O(a) sr(a) costuma comer saladas cruas todos ou quase todos os dias? O(a) sr(a) costuma comer verduras e legumes cozidos todos ou quase todos os dias?". As demais variáveis referentes a hábitos alimentares de risco foram possíveis pelas questões: "Em quantos dias da semana o(a) sr(a) toma refrigerante? Com que frequência o(a) sr(a) costuma ingerir alguma bebida alcoólica? Num único dia o sr (para homem) chega a tomar mais do que duas latas de cerveja ou mais do que duas taças de vinho ou mais do que duas doses de qualquer outra bebida alcoólica? ou Num único dia a sra chega a tomar mais do que uma lata de cerveja, ou mais do que uma taça de vinho, ou mais do que uma dose de qualquer outra bebida alcoólica? $\mathrm{O}(\mathrm{a}) \mathrm{sr}(\mathrm{a})$ costuma tomar leite? Quando o(a) sr(a) toma leite, que tipo de leite costuma tomar? O(a) $\mathrm{sr}(\mathrm{a})$ costuma comer carne vermelha, por exemplo boi, porco, cabrito? Quando o(a) sr(a) come carne vermelha com gordura, o(a) sr(a) costuma tirar o excesso de gordura ou comer com a gordura? O(a) sr(a) costuma comer frango? Quando o(a) sr(a) come frango com pele, o(a) sr(a) costuma tirar a pele ou comer com a pele?". Não se considerou o tamanho das porções, mas sim o hábito como marcador de consumo inadequado.

A idade foi categorizada em seis grupos etários: 18-24, 25-34, 35-44, 45-54, 55-64 ou $\geq$ 65 anos; a escolaridade em quatro grupos: 0-4, 5-8, 9-11 ou $\geq 12$ anos de estudo; a situação conjugal estável em dois grupos: não (sem companheiro) ou sim (com companheiro).

Para verificar diferença entre os sexos e associação entre excesso de peso e as demais variáveis, foi utilizado o teste do qui-quadrado. As variáveis associadas com excesso de peso, ao nível $p<0,05$, foram selecionadas para análise multivariada, por regressão logística, considerando-se o consumo de feijão $(\geq 5,3$ a 4 ou $\leq 2$ dias por semana) como variável explanatória e as demais variáveis como de confusão, sendo as variáveis com $0,05 \leq p<0,20$ utilizadas para ajuste do modelo com a finalidade de aumentar a robustez do estudo.

Adicionalmente, teste de associação pelo qui-quadrado foi realizado para avaliar as frequências de consumo de feijão ( $\geq 5,3$ a 4 ou $\leq 2$ dias por semana) com as demais variáveis, bem como regressão logística para identificar os preditores do consumo de feijão $\geq 5$ dias por semana.

Os dados foram analisados separadamente para cada sexo, considerando-se intervalo de confiança de 95\% e nível de significância de 5\%, com o auxílio do programa Statistical Package for the Social Science (SPSS) versão 13.0.

Neste estudo, por se tratar de entrevista por telefone, o consentimento livre e esclarecido foi substituído pelo consentimento verbal obtido por ocasião dos contatos telefônicos com os entrevistados, sendo esclarecidas a possibilidade de desistir de participar do estudo a qualquer momento, a inexistência de riscos ou danos à saúde e a garantia do sigilo das informações obtidas, a serem utilizadas apenas para fins de pesquisa. Este estudo faz parte do projeto "SIMTEL: Cinco cidades: implantação, avaliação e resultados de um sistema municipal de monitoramento de fatores de risco nutricionais para doenças crônicas não transmissíveis a partir de entrevistas telefônicas em cinco municípios brasileiros", que foi submetido e aprovado pelo Comitê de Ética da Faculdade de Saúde Pública da Universidade de São Paulo (protocolo $n^{\circ} 1225$ ).

\section{RES ULTADOS}

O estudo compreendeu 2352 indivíduos (39,8\% do sexo masculino e $60,2 \%$ do feminino). 
A Tabela 1 mostra que não houve diferença entre os sexos quanto à escolaridade e à situação conjugal, todavia observou-se maior percentual de homens mais jovens comparativamente às mulheres. O excesso de peso foi maior para os homens, assim como a prática de atividade física no lazer, o hábito de baixo consumo de frutas e hortaliças, de alto consumo de refrigerante, de bebida alcoólica e carne com gordura ou frango com pele. As mulheres apresentaram maior frequência de baixo consumo de feijão.
A Tabela 2 mostra que o excesso de peso, nos homens, foi maior para: os mais velhos, os de maior escolaridade, os com situação conjugal estável, os que consomem menos feijão, os que não têm hábito de consumir leite integral e carne com gordura ou frango com pele. Entre as mulheres, a associação do excesso de peso deu-se diretamente com a idade e inversamente com anos de estudo. Foi maior para as mulheres com união conjugal estável, para as que consumiram menos feijão e para as que não têm hábito de consumir

Tabela 1. Distribuição da população adulta ( $\geq 18$ anos) com telefone, segundo características sociodemográficas, excesso de peso, atividade física no lazer e características do consumo alimentar por sexo. Belém (PA), 2005.

\begin{tabular}{|c|c|c|c|c|c|c|}
\hline \multirow{2}{*}{ Características } & \multicolumn{3}{|c|}{ Masculino $(n=936)$} & \multicolumn{2}{|c|}{ Feminino $(n=1416)$} & \multirow{2}{*}{$p$} \\
\hline & $\%$ & IC & $95 \%$ & $\%$ & $95 \%$ & \\
\hline Idade (anos) & & & & & & $<0,010$ \\
\hline 18 a 24,99 & 23,0 & $(20,3$ & $-25,8)$ & 15,6 & $(13,8-17,6)$ & \\
\hline 25 а 34,99 & 25,9 & $(23,1$ & - 28,8) & 24,6 & $(22,4-26,9)$ & \\
\hline 35 а 44,99 & 21,8 & $(19,2$ & $-24,6)$ & 23,0 & $(20,8-25,3)$ & \\
\hline 45 a 54,99 & 12,7 & $(10,7$ & $-15,1)$ & 17,2 & $(15,3-19,3)$ & \\
\hline 55 a 64,99 & 8,7 & $(7,0$ & $-10,7)$ & 10,8 & $(9,3-12,6)$ & \\
\hline$\geq 65$ & 8,0 & $(6,4$ & $-10,0)$ & 8,8 & $(7,4-10,5)$ & \\
\hline Anos de estudo & & & & & & 0,350 \\
\hline 0 a 4 & 10,1 & $(8,3$ & $-12,3)$ & 10,4 & $(8,9-12,1)$ & \\
\hline 5 a 8 & 19,1 & $(16,7$ & $-21,8)$ & 16,5 & $(14,6-18,5)$ & \\
\hline 9 a 11 & 48,0 & $(44,7$ & $-51,2)$ & 50,8 & $(48,2-53,5)$ & \\
\hline$\geq 12$ & 22,8 & $(20,1$ & $-25,6)$ & 22,3 & $(20,2-24,6)$ & \\
\hline Situação conjugal estável & & & & & & 0,060 \\
\hline Não & 41,0 & $(37,9$ & $-44,3)$ & 37,2 & $(34,7-39,8)$ & \\
\hline $\operatorname{Sim}$ & 59,0 & $(55,7$ & $-62,1)$ & 62,8 & $(60,2-65,3)$ & \\
\hline Excesso de peso & & & & & & $<0,010$ \\
\hline Não & 50,7 & $(47,5$ & $-54,0)$ & 66,0 & $(63,4-68,4)$ & \\
\hline $\operatorname{Sim}$ & 49,3 & $(46,0$ & $-52,5)$ & 34,0 & $(31,6-36,6)$ & \\
\hline Atividade física no lazer & & & & & & $<0,010$ \\
\hline Não & 42,9 & (39, & $8-46,2)$ & 64,1 & $(61,6-66,6)$ & \\
\hline $\operatorname{Sim}$ & 57,1 & $(53$, & $8-60,2)$ & 35,9 & $(33,4-38,4)$ & \\
\hline Consumo de feijão (dias por semana) & & & & & & $<0,010$ \\
\hline$\geq 5$ & 52,5 & $(49,2$ & $-55,7)$ & 32,7 & $(30,3-35,2)$ & \\
\hline 3 a 4 & 21,0 & $(18,5$ & - 23,8) & 24,2 & $(22,0-26,6)$ & \\
\hline$\leq 2$ & 26,5 & $(23,7$ & $-29,5)$ & 43,1 & $(40,5-45,7)$ & \\
\hline Consumo de frutas e hortaliças $<5$ dias por semana & 84,9 & $(82,4$ & $-87,1)$ & 77,5 & $(75,2-79,6)$ & $<0,010$ \\
\hline Consumo de refrigerante $\geq 3$ dias por semana & 42,4 & $(39,2$ & $-45,7)$ & 24,2 & $(22,0-26,6)$ & $<0,010$ \\
\hline $\begin{array}{l}\text { Consumo de bebida alcoólica }>2 \text { doses por dia para homem e }>1 \\
\text { para mulher }\end{array}$ & 42,8 & $(39,7$ & $-46,1)$ & 11,9 & $(10,3-13,8)$ & $<0,010$ \\
\hline Hábito de consumo de leite integral & 67,4 & $(64,3$ & $-70,4)$ & 64,4 & $(61,8-66,9)$ & 0,133 \\
\hline Hábito de consumo de carne com gordura ou frango com pele & 39,6 & $(36,5$ & $-42,9)$ & 21,5 & $(19,4-23,7)$ & $<0,010$ \\
\hline
\end{tabular}

Fonte: Simtel ${ }^{2}$. 
leite integral. Embora, para mulheres, consumo de feijão e de leite integral apresentem associação com excesso de peso $(p<0,01)$, a significância para o consumo de feijão foi muito maior ( $p=0,00003$ ) do que para leite $(0,006)$. Assim, tanto para homens como para mulheres, o consumo de feijão foi a variável de consumo alimentar que melhor se associou com excesso de peso.

As variáveis que se associaram com excesso de peso ao nível de $p<0,20$ foram incluídas no modelo de regressão logística para o cálculo das razões de chance de excesso de peso (Tabelas 3

Tabela 2. Prevalência de excesso de peso na população adulta ( $\geq 18$ anos) com telefone, segundo características sociodemográficas, atividade física no lazer e de consumo alimentar. Belém (PA), 2005.

\begin{tabular}{|c|c|c|c|c|}
\hline \multirow[b]{2}{*}{ Características } & \multicolumn{2}{|c|}{ Masculino } & \multicolumn{2}{|c|}{ Feminino } \\
\hline & $\%$ & $p$ & $\%$ & $p$ \\
\hline Idade (anos) & & $<0,010$ & & $<0,010$ \\
\hline 18 a 24,99 & 27,4 & & 10,9 & \\
\hline 25 a 34,99 & 46,3 & & 24,4 & \\
\hline 35 a 44,99 & 61,8 & & 37,2 & \\
\hline 45 a 54,99 & 61,3 & & 41,8 & \\
\hline 55 a 64,99 & 64,2 & & 57,5 & \\
\hline$\geq 65$ & 52,0 & & 49,6 & \\
\hline Anos de estudo & & $<0,010$ & & $<0,010$ \\
\hline 0 a 4 & 56,8 & & 51,0 & \\
\hline 5 a 8 & 53,1 & & 39,9 & \\
\hline 9 a 11 & 41,6 & & 31,5 & \\
\hline$\geq 12$ & 58,7 & & 27,5 & \\
\hline Situação conjugal estável & & $<0,010$ & & $<0,010$ \\
\hline Não & 35,2 & & 25,2 & \\
\hline $\operatorname{Sim}$ & 59,1 & & 39,3 & \\
\hline Atividade física no lazer & & 0,047 & & 0,477 \\
\hline Não & 46,2 & & 62,9 & \\
\hline Sim & 53,8 & & 37,1 & \\
\hline Consumo de feijão (dias por semana) & & $<0,010$ & & $<0,010$ \\
\hline$\geq 5$ & 44,4 & & 27,69 & \\
\hline 3 a 4 & 51,8 & & 31,2 & \\
\hline$\leq 2$ & 56,9 & & 40,5 & \\
\hline Consumo de frutas e hortaliças $<5$ dias por semana & & 0,935 & & 0,320 \\
\hline Não & 48,9 & & 36,4 & \\
\hline Sim & 49,3 & & 33,4 & \\
\hline Consumo de refrigerante $\geq 3$ dias por semana & & 0,323 & & 0,053 \\
\hline Sim & 51,1 & & 29,7 & \\
\hline Não & 47,9 & & 35,4 & \\
\hline Consumo de bebida alcoólica $>2$ doses por dia para homem e $>1$ para mulher & & 0,055 & & 0,662 \\
\hline Não & 46,5 & & 34,2 & \\
\hline $\operatorname{Sim}$ & 52,9 & & 32,5 & \\
\hline Hábito de consumo de leite integral & & 0,028 & & $<0,010$ \\
\hline Não & 54,4 & & 38,7 & \\
\hline $\operatorname{sim}$ & 46,8 & & 31,5 & \\
\hline Hábito de consumo de carne com gordura ou frango com pele & & 0,036 & & 0,454 \\
\hline Não & 52,0 & & 34,5 & \\
\hline Sim & 45,0 & & 32,2 & \\
\hline
\end{tabular}

Fonte: Simtel ${ }^{2}$ 
e 4). Para os homens, compuseram o modelo: idade, escolaridade, situação conjugal estável, atividade física no lazer, consumo de feijão, hábito de consumo de leite integral e de carne com excesso de gordura, mas, após ajuste para todas as variáveis, permaneceu apenas situação conjugal estável e consumo de feijão. Homens com situação conjugal estável têm 1,6 vezes mais chance de terem excesso de peso do que aqueles sem situação conjugal estável, mesmo após ajuste para idade; e aqueles com baixo consumo de feijão ( $\leq 2$ dias por semana) têm 1,4 vez mais chance de terem excesso de peso do que os com alto consu- mo ( $\geq 5$ dias por semana). Para as mulheres, compuseram o modelo: idade, escolaridade, situação conjugal estável, consumo de feijão e hábito de consumo de leite integral, tendo permanecido, após ajuste para todas as variáveis, no modelo idade, escolaridade e consumo de feijão. Mulheres entre 25 e 44 anos de idade têm menor chance de excesso de peso do que as demais, assim como aquelas com consumo de feijão menor do que cinco dias por semana. Mulheres com escolaridade entre cinco e oito anos de estudo têm quase o dobro de chance de excesso de peso do que as demais.

Tabela 3. Razão de chance (Odds Ratio - OR) de excesso de peso em homens ( $\geq 18$ anos) com telefone, segundo características sociodemográficas, atividade física no lazer e de consumo alimentar. Belém (PA), 2005.

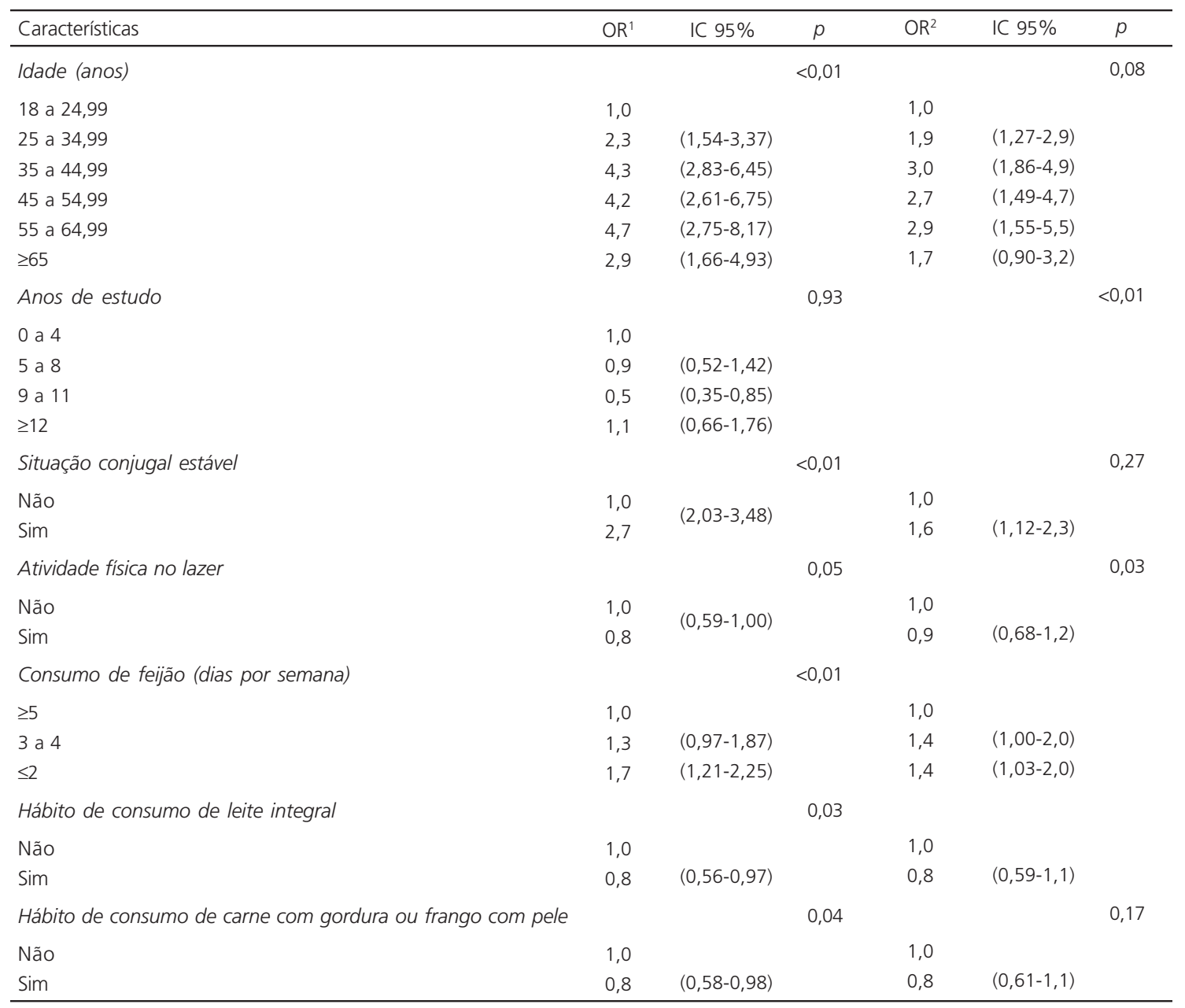

OR': Odds ratio bruto; OR2: Odds ratio ajustado

FONTE: SIMTEL 2 . 
246 | S.A. SILVA et al.

Tabela 4. Razão de chance (Odds Ratio - OR) de excesso de peso em mulheres ( $\geq 18$ anos) com telefone, segundo características sociodemográficas, atividade física no lazer e de consumo alimentar. Belém (PA), 2005.

\begin{tabular}{|c|c|c|c|c|c|c|}
\hline Características & $\mathrm{OR}^{1}$ & IC 95\% & $p$ & $\mathrm{OR}^{2}$ & IC 95\% & $p$ \\
\hline Idade (anos) & & & $<0,01$ & & & $<0,01$ \\
\hline 18 a 24,99 & 1,0 & & & 1,0 & & \\
\hline 25 а 34,99 & 2,7 & $(1,63-4,33)$ & & 0,2 & $(1,10-0,32)$ & \\
\hline 35 а 44,99 & 4,9 & $(3,01-7,87)$ & & 0,4 & $(0,27-0,70)$ & \\
\hline 45 a 54,99 & 5,9 & $(3,60-9,67)$ & & 0,8 & $(0,48-1,20)$ & \\
\hline 55 а 64,99 & 11,1 & $(6,53-18,9)$ & & 0,9 & $(0,56-1,41)$ & \\
\hline$\geq 65$ & 8,1 & $(4,66-14,0)$ & & 1,5 & $(0,91-2,41)$ & \\
\hline Anos de estudo & & & $<0,01$ & & & 0,04 \\
\hline 0 a 4 & 1,0 & & & & & \\
\hline 5 a 8 & 0,6 & $(0,42-0,97)$ & & 1,0 & $(1,23-3,08)$ & \\
\hline 9 a 11 & 0,4 & $(0,31-0,63)$ & & 2,0 & $(0,93-2,04)$ & \\
\hline$\geq 12$ & 0,4 & $(0,24-0,55)$ & & 1,4 & $(0,96-1,78)$ & \\
\hline Situação conjugal estável & & & $<0,01$ & & & 0,06 \\
\hline Não & 1,0 & & & 1,0 & & \\
\hline Sim & 1,9 & $(1,51-2,43)$ & & 0,8 & $(0,67-1,15)$ & \\
\hline Consumo de feijão (dias por semana) & & & $<0,01$ & & & $<0,01$ \\
\hline$\geq 5$ & 1,0 & & & 1,0 & & \\
\hline 3 a 4 & 1,2 & $(0,87-1,61)$ & & 0,6 & $(0,44-0,77)$ & \\
\hline$\leq 2$ & 1,8 & $(1,37-2,31)$ & & 0,7 & $(0,55-0,99)$ & \\
\hline Hábito de consumo de leite integral & & & $<0,01$ & & & $<0,16$ \\
\hline Não & 1,0 & & & 1,0 & & \\
\hline Sim & 0,7 & $(0,58-0,91\})$ & & 1,2 & $(0,96-1,57)$ & \\
\hline
\end{tabular}

OR': Odds ratio bruto; OR2: Odds ratio ajustado FONTE: SIMTEL ${ }^{2}$.

Com a análise multivariada, demonstrou-se que o risco do excesso de peso aumenta com a redução do consumo de feijão para ambos os sexos. Para homens após ajuste para idade, escolaridade, situação conjugal estável, atividade física no lazer, hábito de consumo de leite integral e de carne com excesso de gordura o risco de excesso de peso é cerca de 1,4 vez maior entre os que consomem feijão menos do que cinco vezes na semana. Para mulheres, ajustado para idade, escolaridade, situação conjugal estável, hábito de consumo de leite integral, é aproximadamente 0,3 vez menor para as que consomem feijão menos do que três vezes por semana.

A Tabela 5 mostra que o consumo de feijão é maior para os homens mais jovens, sem situação conjugal estável, sem excesso de peso, com hábito de consumo de leite integral e de carne com excesso de gordura. Para as mulheres, foi maior entre as mais jovens, com baixa escolaridade, sem excesso de peso, insuficientemente ativas no lazer, sem consumo de risco de bebidas alcoólicas e com hábito de consumo de leite integral. A análise por regressão logística, incluindo todas as variáveis acima, mostra que, para os homens, apenas o não excesso de peso se manteve associado com o alto consumo de feijão ( $\geq 5$ dias por semana); e, para as mulheres, todas as variáveis foram mantidas (dados não mostrados).

\section{I S C U S S Ã O}

O excesso de peso atinge cerca de um bilhão de adultos no mundo e corresponde a um fator de risco relevante para o surgimento de doenças crônicas ${ }^{1}$. Inquéritos nacionais recentes apontam para o aumento do excesso de peso na 
Tabela 5. Distribuição (\%) de indivíduos ( $\geq 18$ anos) com telefone conforme frequência de consumo de feijão, segundo características sociodemográficas, excesso de peso, atividade física no lazer e demais variáveis de consumo alimentar por sexo. Belém (PA), 2005.

\begin{tabular}{|c|c|c|c|c|c|c|c|c|}
\hline \multirow{3}{*}{ Características } & \multicolumn{8}{|c|}{ Consumo de feijão (dias por semana) } \\
\hline & \multicolumn{4}{|c|}{ Homens (\%) } & \multicolumn{4}{|c|}{ Mulheres (\%) } \\
\hline & $\geq 5$ & 3 a 4 & $\leq 2$ & $p$ & $\geq 5$ & 3 a 4 & $\leq 2$ & $p$ \\
\hline Idade (anos) & & & & $<0,01$ & & & & $<0,01$ \\
\hline 18 a 24,99 & 54,9 & 26,9 & 18,1 & & 40,3 & 24,0 & 35,7 & \\
\hline 25 a 34,99 & 56,2 & 20,6 & 23,1 & & 31,0 & 30,5 & 38,5 & \\
\hline 35 a 44,99 & 52,0 & 21,6 & 26,5 & & 31,7 & 23,1 & 45,2 & \\
\hline 45 a 54,99 & 52,1 & 17,6 & 30,3 & & 33,2 & 19,3 & 47,5 & \\
\hline 55 a 64,99 & 46,9 & 14,8 & 38,3 & & 27,5 & 22,2 & 50,3 & \\
\hline$\geq 65$ & 41,3 & 16,0 & 42,7 & & 32,0 & 22,4 & 45,6 & \\
\hline Anos de estudo & & & & 0,10 & & & & $<0,01$ \\
\hline 0 a 4 & 51,6 & 17,9 & 30,5 & & 42,2 & 19,7 & 38,1 & \\
\hline 5 a 8 & 52,0 & 21,8 & 26,3 & & 36,1 & 21,9 & 42,1 & \\
\hline 9 a 11 & 54,3 & 23,2 & 22,5 & & 33,8 & 24,0 & 42,2 & \\
\hline$\geq 12$ & 49,3 & 17,4 & 33,3 & & 23,4 & 28,5 & 48,1 & \\
\hline Situação conjugal estável & & & & 0,03 & & & & 0,10 \\
\hline Não & 56,5 & 21,4 & 22,1 & & 29,2 & 25,8 & 45,0 & \\
\hline Sim & 49,6 & 20,8 & 29,5 & & 34,8 & 23,3 & 42,0 & \\
\hline Excesso de peso & & & & $<0,01$ & & & & $<0,01$ \\
\hline Não & 57,5 & 20,0 & 22,5 & & 35,9 & 25,3 & 38,9 & \\
\hline Sim & 47,3 & 22,1 & 30,6 & & 26,6 & 22,2 & 51,2 & \\
\hline Atividade física no lazer & & & & 0,06 & & & & 0,03 \\
\hline Não & 48,5 & 21,4 & 30,1 & & 35,1 & 23,7 & 41,2 & \\
\hline Sim & 55,4 & 20,8 & 23,8 & & 28,3 & 25,2 & 46,5 & \\
\hline Consumo de frutas e hortaliças $<5$ dias por semana & & & & 0,63 & & & & 0,42 \\
\hline Não & 50,4 & 19,9 & 29,8 & & 35,7 & 22,9 & 41,4 & \\
\hline Sim & 52,8 & 21,3 & 25,9 & & 31,8 & 24,6 & 43,6 & \\
\hline Consumo de refrigerante $\geq 3$ dias por semana & & & & 0,55 & & & & 0,80 \\
\hline Sim & 51,4 & 22,3 & 26,3 & & 32,1 & 23,3 & 44,6 & \\
\hline Não & 53,9 & 19,4 & 26,7 & & 32,9 & 24,5 & 42,6 & \\
\hline $\begin{array}{l}\text { Consumo de bebida alcoólica }>2 \text { doses por dia para homem e } \\
>1 \text { para mulher }\end{array}$ & & & & 0,22 & & & & 0,03 \\
\hline Não & 50,5 & 20,9 & 28,6 & & 33,8 & 24,3 & 41,9 & \\
\hline Sim & 55,1 & 21,2 & 23,7 & & 24,9 & 23,7 & 51,5 & \\
\hline Hábito de consumo de leite integral & & & & $<0,01$ & & & & $<0,01$ \\
\hline Não & 47,2 & 19,0 & 33,8 & & 26,0 & 23,4 & 50,6 & \\
\hline Sim & 55,0 & 22,0 & 23,0 & & 36,4 & 24,7 & 38,9 & \\
\hline Hábito de consumo de carne com gordura ou frango com pele & & & & 0,03 & & & & 0,48 \\
\hline Não & 49,7 & 20,7 & 29,6 & & 31,9 & 24,6 & 43,4 & \\
\hline $\mathrm{Sim}$ & 56,6 & 21,6 & 21,8 & & 35,5 & 22,7 & 41,8 & \\
\hline
\end{tabular}

Fonte: Simtel ${ }^{2}$.

população brasileira. Em Belém, o estudo do Instituto Nacional do Câncer (INCA) ${ }^{15}$ - mostrou uma prevalência de excesso de peso de 39,5\% em homens e $30,6 \%$ em mulheres. Dados de $2006^{2}$ apontam em Belém 47,8\% e 30,9\% de excesso de peso, respectivamente em homens e mulheres, mostrando um claro aumento para os homens. Nossos dados revelam 49,3\% de excesso de peso 
para os homens e $34,0 \%$ para as mulheres, valores semelhantes aos de 2006, considerando-se o intervalo de confiança.

Neste estudo, a prevalência de excesso de peso foi obtida pelo cálculo de peso e altura referidos pelo entrevistado, procedimento usualmente adotado em inquéritos epidemiológicos. Segundo Peixoto et al. ${ }^{16}$, em estudo sobre a validade do peso e da altura autorreferidos realizado na cidade de Goiânia, observou-se alta concordância entre as medidas de peso reportado e aferido e entre altura reportada e referida em inquérito de base populacional, permitindo sua utilização em estudos de prevalência e monitoramento. O estudo aponta que os dados podem ser coletados por meio de entrevistas frente a frente, envio de questionários pelo correio ou entrevistas por telefone; procedimentos que permitem avaliar e acompanhar ao longo do tempo amostras representativas da população, com menor custo e simplificação do trabalho de campo. Em inquérito realizado por entrevistas telefônicas para monitorar fatores de risco para doenças crônicas, Monteiro et al. ${ }^{17}$ indicaram confiabilidade das estimativas obtidas, maior agilidade e menor custo quando comparadas a levantamentos com coleta de dados em domicílio, apontando esse método como uma promissora alternativa para a obtenção de informações sobre fatores de risco para doenças crônicas não transmissíveis em nosso meio, além de terem validado inquérito para estimativa do padrão alimentar ${ }^{17}$.

Gigante et al. ${ }^{18}$, analisando dados da Pesquisa Nacional de Saúde e Nutrição de 1989, observaram que a ocorrência de obesidade aumenta diretamente com a idade em ambos os sexos. O estudo do INCA ${ }^{15}$ revela maior prevalência de excesso de peso nos indivíduos com mais de 50 anos, comparativamente aos mais jovens. Neste estudo, o aumento do excesso de peso também esteve associado com o aumento da idade em homens e mulheres, observando-se aumento até 64 anos de idade e ligeira queda a partir daí, igualmente aos dados obtidos pelo sistema nacional de vigilância de fatores de risco e proteção para doenças crônicas não transmissíveis por entrevista telefônica Vigilância de Fatores de Risco e Proteção para Doenças Crônicas por Inquérito Telefônico (Vigitel)².

Quanto à escolaridade, nossos dados apontam queda da prevalência de excesso de peso com o aumento da escolaridade em ambos os sexos, porém com aumento na faixa de maior escolaridade no sexo masculino, semelhante aos dados do VIGITEL ${ }^{2}$. Em estudo de base populacional realizado em 2003, em São Leopoldo (RS), com mulheres, também foi identificada a escolaridade como variável de proteção para o excesso de peso ${ }^{19}$.

Embora os homens apresentem mais excesso de peso do que as mulheres, eles também são mais ativos no lazer, consomem mais feijão, bebidas alcoólicas, refrigerantes, carne com gordura ou frango com pele, além de menor consumo de frutas, verduras e legumes. Todavia essas variáveis estão inter-relacionadas. Daí, a necessidade da análise bivariada (excesso de peso versus demais variáveis) para seleção das variáveis possivelmente explanatórias do excesso de peso, que serão utilizadas na análise multivariada, para identificar, de fato, aquelas relacionadas ao excesso de peso.

Além da idade e da escolaridade, observamos associação entre excesso de peso e estado marital estável, mas que se mantém, após ajuste, apenas para os homens, mostrando-se como fator de confundimento para as mulheres, uma vez que também há a interferência da idade. Entre as variáveis de consumo alimentar, associaram-se com excesso de peso, o menor consumo de feijão e o não consumo de leite integral em ambos os sexos e o não consumo de carne com gordura ou frango com pele no sexo masculino, sendo o consumo de feijão o mais significativo.

Por se tratar de estudo transversal, tanto desfecho como variável explanatória são medidas ao mesmo tempo, de modo que as associações entre excesso de peso e não consumo de leite integral e não consumo de carne com gordura ou frango com pele podem ser consideradas como 
causalidade reversa, isto é, indivíduos com excesso de peso passam a ter mais cuidado com a dieta na busca de um estado nutricional adequado, assim como a prática do exercício físico no lazer.

Neste estudo, o risco de excesso de peso em homens foi cerca de $40 \%$ maior naqueles com consumo de feijão menor do que cinco vezes na semana e em mulheres foi cerca de 30\% menor naquelas com consumo menor do que três vezes na semana.

Após controle das variáveis sociodemográficas, fica evidente o papel de proteção do feijão para o melhor estado nutricional dos homens, ainda que também se associe com o hábito de consumo de leite integral e de carne com excesso de gordura. O papel protetor do consumo de feijão em relação ao excesso de peso foi constatado por Sichieri ${ }^{7}$, ao analisar a dieta brasileira. O consumo de feijão tem sido associado a um hábito alimentar mais sadio, sendo recomendado o consumo de pelo menos uma porção ao dia, conforme o Guia Alimentar para a População Brasileira ${ }^{20}$. Em estudo realizado em São Paulo sobre consumo de alimentos de risco e proteção para doenças cardiovasculares ${ }^{21}$, aplicando-se questionário de frequência alimentar, observou-se que o consumo diário de feijão atingiu 55,8\% da população estudada. No conjunto, o consumo de alimentos de risco (alimentos e/ou preparações fontes de gorduras saturadas e/ou colesterol e/ou ácidos graxos trans e/ou sódio e/ou glicídios, como carnes, ovos, leite e derivados, doces, frituras e massas) foi estatisticamente maior entre indivíduos de menor escolaridade.

Este estudo limita-se à população com telefone fixo em sua residência, mas permite análise de associação, embora não seja o modelo adequado para se avaliar causalidade e efeito, por se tratar de estudo transversal, isto é, as variáveis são coletadas simultaneamente. Nesse sentido, novos estudos mais controlados, que avaliem causa-efeito, são necessários para melhor entendimento da associação entre consumo de feijão e excesso de peso.

\section{COLABORADORES}

S.A. SILVA e P.N.S. SANTOS participaram da coleta de dados e da redação do artigo. E.C. MOURA idealizou o estudo, orientou a análise de dados e revisou o artigo.

\section{REFERÊ NCIAS}

1. World Health Organization. Global strategy on diet, physical activity and health. Geneva: WHO; 2002.

2. Brasil. Ministério da Saúde. Vigilância de Fatores de Risco e Proteção para Doenças Crônicas por Inquérito Telefônico. Brasília: Ministério da Saúde; 2007.

3. Instituto Brasileiro de Geografia e Estatística. Pesquisa de orçamentos familiares 2002-2003: primeiros resultados: Brasil e grandes regiões. Rio de Janeiro: IBGE; 2004.

4. Malta DC, Cezário AC, Moura L, Morais Neto OL, Silva Jr JB. Construção da vigilância e prevenção das doenças crônicas não transmissíveis no contexto do sistema único de saúde. Epidemiol Serv Saúde. 2006; 15:47-64.

5. Schulze MB, Manson JE, Ludwing DS, Colditz GA, Stampfer MJ, Willet WC, et al. Sugar-sweetened beverages, weght gain, and incidence of type 2 diabetes in young and middle-aged women. Jama. 2004; 292(8):927-34.

6. Arif AA, Rohrer JE. Patterns of alcohol drinking and its association with obesity: data from the third national health and nutrition examination survey 1988-1994. BMC Public Health. 2005; 5:126. doi:10.1186/1471-2458-5-126.

7. Sichieri R. Dietary patterns and their associations with obesity In the Brazilian city of Rio de Janeiro. Obes Res. 2002; 10(1):42-9.

8. Mondini L, Monteiro CA. Mudanças no padrão de alimentação da população urbana brasileira (1962-1988). Rev Saúde Pública. 1994; 28 (6): 433-9. doi: 10.1590/S0034-89101994000600 007.

9. Monteiro CA, Mondini L, Costa RBL. Mudanças na composição e adequação nutricional da dieta familiar nas áreas metropolitanas do Brasil (1988-1996). Rev Saúde Pública. 2000; 34(3): 251-8. doi: 10.1590/S0034-89102000000300 007.

10. Jaime PC, Monteiro CA. Fruit and vegetable intake by Brazilian adults, 2003. Cad Saúde Pública. 2005; 21 (Sup):S19-S24. doi: 10.1590/S0102-311X2005 000700003. 
11. Levy-Costa RB, Sichieri R, Pontes NS, Monteiro CA. Disponibilidade domiciliar de alimentos no Brasil: distribuição e evolução (1974-2003). Rev Saúde Pública. 2005; 39(4):530-40. doi: 10.1590/\$0034-8 9102005000400003.

12. Monteiro CA, Moura EC, Jaime PC, Lucca A, Florindo AA, Figueiredo ICR, et al. Monitoramento de fatores de risco para doenças crônicas por meio de entrevistas telefônicas: métodos e resultados no Município de São Paulo. Rev Saúde Pública. 2005; 39(1):47-57. doi: 10.1590/S0034-891020 05000100007.

13. Word Health Organization. Physical status: the use and interpretation of antropometry. Geneva: WHO;1995. WHO Technical Report, Series 854.

14. Monteiro CA, Florindo AA, Claro RM, Moura EC. Validade de indicadores de atividade física e sedentarismo obtidos por inquérito telefônico. Rev Saúde Pública. 2008; 42(4)575-81. doi: 10.1590/S0 034-89102008000400001.

15. Brasil. Ministério da Saúde. Secretaria de Vigilância em Saúde. Inquérito domiciliar sobre comportamentos de risco e morbidade referida de doenças e agravos não transmissíveis: Brasil, 15 capitais e Distrito Federal, 2002-2003. Rio de Janeiro: INCA; 2004.

16. Peixoto MRG, Benício MHA, Jardim PCBV. Validade do peso e da altura auto-referidos: estudo de
Goiânia. Rev Saúde Pública. 2006; 40(6):1065-72. doi: 10.1590/S0034-89102006000700015.

17. Monteiro CA, Moura EC, Jaime PC, Claro RC. Validade de indicadores do consumo de alimentos e bebidas obtidos por inquérito telefônico. Rev Saúde Pública. 2008; 42(4):582-89. doi: 10.1590/S0 034-89102008000400002.

18. Gigante DP, Barros FC, Post CLA, Olinto MTA. Prevalência de obesidade em adultos e seus fatores de risco. Rev Saúde Pública. 1997, 31(3):236-46. doi: 10.1590/S0034-89101997000300004.

19. Teichmann L, Olinto MTA, Costa JSD, Ziegler D. Fatores de risco associados ao sobrepeso e a obesidade em mulheres de São Leopoldo, RS. Rev Bras Epidemiol. 2006; 9(3):360-73. doi: 10.1590/S1 415-790X2006000300010.

20. Brasil. Ministério da Saúde. Secretaria de Atenção à Saúde. Guia alimentar para população brasileira: Promovendo a alimentação saudável. Brasília: Ministério da Saúde; 2006.

21. Neumann AICP, Shirassu MM, Fisberg RM. Consumo de alimentos de risco e proteção para doenças cardiovasculares entre funcionários públicos. Rev Nutr. 2006; 19(1):19-28. doi: 10.1590/\$1415-527 32006000100002 .

Recebido em: 29/7/2008

Versão final reapresentada em: 29/4/2009 Aprovado em: 6/10/2009 